by which the bones had united has seemed to "give way," this has been due not to any defect in the uniting substance, but to the fact that the upper fragment having become firmly fixed to the neighbouring bone the stretching of the uniting material was a necessary preliminary to obtaining of any movement in the joint at all. It, in fact, became apparent that by far the most important point in the treatment of these frac. tures was to prevent the fixation of the upper fragment. Bony union is a good thing no doubt, and there is an evident loss of mechanical efficiency in a loose and elongated fibrous junction; but no amount of perfection in the union of the fractured surfaces is of much utility, so long as the upper fragment is held fast and blocks the way for the transmission of power from the contracting muscle to the distal portion of the limb. Hence, for some years past, surgeons having seen what are the real obstacles to success in the treatment of the patella or olecranon, have devoted themselves far more than they did in old days to maintaining mobility in the joint, and above all things to preventing that fixation of the upper fragment which is the source and origin of most of the difficulty in restoring the functions of the limb. Speaking on this subject recently at St. George's Hospital, Mr. William Bennett said that in a case of fracture of the patella, in the absence of the treatment by suture now so commonly employed, the final result was dependent mainly upon two points, (1) the amount of laceration of the lateral expansions about the knee at the time of injury (the less these expansions are ruptured the more being the strength left in the joint) ; and (2) the amount of mobility finally retained by the upper fragment of the patella. If the fragment does not contract inveterate adhesions, and if it fin ally becomes freely moveable on the femur, a more or less useful limb follows, the strength of the limb as a rule being in inverse proportion to the length of the uniting medium. If, on the other hand, fixation of this upper fragment occurs, then, however good the union, the interference with the mobility and strength of the joint is so great that the patient is greatly crippled. The first object then in the treatment of a case of fracture of the patella, whether it has been wired or not, is to prevent by constant manipulation any chance of adhesion of the upper fragment to the femur-a line of treatment which excludes any form "of splint which makes the patella inaccessible. This remark, by the by, is not confined to the treatment of fractures, but applies to all cases of inflammation of the knee joint which are liable to be followed by stiffness, in all which cases fixed, immovable apparatus, such as plaster of Paris, silicate of potash, and the like are rendered entirely inapplicable by the fact that they prevent access to the injured part. The best results after fracture of the patella, when not subjected to the wiring treatment, will, he says, be obtained by immediate smooth massage and patella manipulation, followed in a fortnight or earlier by gentle passive movement of the knee; and it is to be remarked how little, if any, stretching of uniting material occurs in cases treated in this way, if only care be taken from the outset to secure free movement of the patella upon the femur. And what is true of the patella is true also in regard to the olecranon. In cases of fracture of this bone, if the case be treated by prolonged immobility, adhesions take place, and the detached fragment of the olecranon becomes practically fixed to the humerus; perhaps not by bony union, but by such strong adhesions that it is very difficult to move it at all; and since, as with the patella, the utility of the limb depends more upon the mobility of the upper fragment than upon the character or apparent strength of the union, it will be found here also, when the fragment is fixed, that on commencing to bend the joint, movement is obtained not by the gliding downwards of the upper fragment, but by a stretching of the union. The fragment remains unmoved, so that, although flexion may be obtained to a great degree, power of extension-that is, of transmitting motive force from the muscle to the ulna-is almost, if not entirely, wanting. Thus the two great cardinal facts to bear in mind in the rational treatment of fracture of the patella and fracture of the olecranon are, first, the avoidance under all circumstances of any chance of the upper part of the patella or of the olecranon becoming adherent to the subjacent bone, which may be prevented by massage and lateral manipulation, combined with gentle flexion and extension; and second, that all manipulation and passive movement should be preceded by smooth rubbing of the muscles by which spasm is removed.

\section{THE ICE.BAG IN THE TREATMENT OF} PNEUMONIA.

IN a clinical lecture on pneumonia, recently delivered at the Liverpool Royal Infirmary, Dr. James Barr spoke highly of the advantages attending the use of the abdominai ice-bag as a means of lowering the temperature, and gave a special warning against the use of coal tar antipyretics for that purpose. He said he had tried the application of the ice-bag to the affected side as recommended by Dr. Lees, but while they afforded great relief to the pleuritic pain they never seemed to control the internal temperature, and he thought that they occasionally lowered the vitality of the part and thus tended towards the development of pneumonia. Ice cradles he regarded as a most unscientific proceeding, for air, being a very bad conductor of heat, its cooling effect was chiefly due to the evaporation produced. Thus a current of moderately warm, dry air would abstract much more heat than a cold atmosphere saturated with moisture, and would certainly be much more agreeable to the patient. The abdomen, however, afforded a large cooling surface, and he thought that the application of one or two ice-bags, sufficient to cover the whole abdomen, would lower the general temperature, and from the action of the cold on the splanchnic area would raise the general arterial pressure, and finally would diminish the frequency and increase the depth of the respirations. Before using this method in pneumonia he had observed its efficacy in a large number of cases of typhoid fever, and he had also observed that except in the groins, which can easily be protected with a little cotton wool, the abdomen is not very sensitive to cold, so that the method was not particularly uncomfortable to the patient. He then related a series of cases so treated with apparently the best results. He insisted, however, on this, that it was in the early stages of the disease that ice was useful. There is no use, he said, in attempting to arrest an inflammation which has reached 
its height. If the lung be in a stage of grey hepatisation, one should encourage resolution by warm poultices. Nor should ice be used in feeble old people, or in those with degenerate hearts, which cannot cope with much peripheral resistance. In very corpulent people, also, ice seemed to fail in removing excess of heat satisfactorily. We should have liked to have heard Dr. Barr's opinion as to the use of Leiter's tube in such cases, this apparatus often being more immediately available than ice, especially in country practice.

\section{EXPANDING MAUSER BULLETS.}

Sir William Srokes, ' writing on the subject of the Mauser bullet and the wounds produced by it, points out that, notwithstanding all that was said in the earlier stages of the war as to the Mauser being a merciful and humane weapon, later experience has gone to show that the injuries produced by it have been of a much more serious character than those at first described. He specially refers to certain remarks made by Sir William MacCormac, Mr. Treves, Mr. Dent, and others who advocate the desirability of "masterly inactivity" in this department of surgery, and goes on to say that his experience in the Maritzburg military hospitals and in the general hospital, Mooi River, compel him to hold opinions which differ largely from the views mentioned. He believes that the gunshot wounds met with in the earlier part of the campaign differed materially in character from those observed of late, and that the increasing gravity of the wounds has been the cause of the increased difficulty which has arisen in keeping them aseptic. This change he attributes to two causes. First, the frequent conversion by the Boers of the Mauser bullet into an expanding one, either by removing a small portion of the case from the apex of the bullet, thus converting it slito a "soft-nosed" one, or by making longitudinal $\mathrm{L}_{\mathrm{ad}}$ round the case, and he adds that when recently at were were adopted by the enemy to increase the gravity of the wounds they inflicted. Secondly, another cause for been greater severity of the wounds observed of late has were that the ranges have been much closer than they closer formerly, it being clearly established that the be. the range the more serious is the injury likely to of a in illustration of his conclusions, he gives details produced of cases in which such grave injuries were of limbs. 'Brit. Med. Jour., June 16.

\section{HYPERTROPHIC PYLORIC STENOSIS IN}

INFANCY.
$D_{R}$. ERIC PRITCHARD ${ }^{1}$ draws attention to this rare publition. So far as he has been able to ascertain, the stenosis records of fatal cases of bypertrophic pyloric by post.morring in infancy, which have been verified a cart.mortem examinations, are 23 in number. After Pritchar review of the cases already published, Dr. The child gives details of a case observed by himself. full time was apparently healthy at birth, was born at had one, of healthy parents, weighed nine pounds, and hand. symptom During the first three weeks there were no ns except sweating. At the third week vomiting set in, the urine was very scanty, and the bowels constipated. Then the child persistently lost weight until at the seventh week it only weighed six pounds. At this time the emaciation was extreme; the tongue and fauces were remarkably clean; the abdomen was flaccid and carinated, and the vertebral column was easily palpated through the abdominal walls; there were no enlarged mesenteric glands; the upper part of the abdomen was more prominent and resonant to percussion; at times the out. line of the stomach was quite evident and extended as far as, and below the umbilicus, and slow waves of contraction could be seen passing from the cardiac end to the pylorus, where, however, no tumour or sense of resistance could be made out. The temperature was normal, but during an attack of convulsions which afterwards occurred, the temperature rose to $104.5 \mathrm{deg}$. F. By aid of nutrient enemata and teaspoonful quantities of food, it seemed to put on a little weight, and romiting ceased, but it died 12 days after it was first seen. At the autopsy the brain and meninges were slightly congested, and the lateral sinus was distended. The œsophagus was distended throughout, " the stomach was enlarged and capable of containing about seven ounces of water. The walls were slightly thickened, especially at the pyloric end. At the pylorus itself was a hard annular thickening, giving almost the appearance of the impaction of some foreign body about the size of a large filbert nut. A No. 5 catheter could just pass through the opening. On splitting the pylorus open the walls were found to be enormously thickened, and to consist of a pale homogeneous tissue." Microscopically there was nothing abnormal in this material. Dr. Pritchard suggests that in these cases the hypertrophy is secondary to over action of the sphincter, that the stenosis is chiefly due to spasm, and that the over action and the inco-ordinated contractions of the sphincter may be due to some fault in the nervous mechanism, although injudicious feeding may be a contributory factor.

$$
1 \text { Archives of Pediatrics, April. }
$$

\section{SECCOTINE IN SURGERY.}

A VERY practical "tip" is given by Dr. James Macaskiel in regard to the extraction of foreign bodies from the ear. He was called to see a schoolboy who had pushed into the meatus a piece of indiarubber which had previously been attached to a lead pencil. The rubber had been driven well in, and as it was an almost exact mould of the passage, and presented a perfectly flat surface, it was impossible to catch hold of it, and syringing did not seem likely to improve matters. Dr. Macaskie therefore cleaned away all the wax with ether and then teased out the end of a small piece of twine, and giving this a good coating of seccotine pushed it tightly against the indiarubber, and packed it closely all round with cotton wool. After this had been left in place for 24 hours there was firm cohesion, and not the slightest difficulty was experienced in withdrawing every. thing en masse. In form this expedient is, we believe, not original. The difficulty has been to obtain a material which would adhere to all sorts of things. For this, and similar purposes, seccotine will be found very useful; but Dr. Macaskie's forethought in cleaning with ether no doubt conduced much to his success.

1 Lancet, June 2. 